\title{
¿Ocean Glider Flight in the Presence of Surface Waves
}

\author{
LuCAS M. MERCKELBACH ${ }^{\mathrm{a}}$ AND JEFFREY R. CARPENTER ${ }^{\mathrm{a}}$

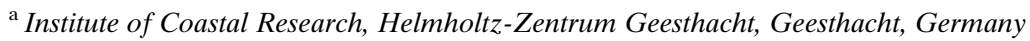

(Manuscript received 16 December 2020, in final form 12 May 2021)

\begin{abstract}
Autonomous, buoyancy-driven ocean gliders are increasingly used as a platform for the measurement of turbulence microstructure. In the processing of such measurements, there is a sensitive (quartic) dependence of the turbulence dissipation rate $\epsilon$ on the speed of flow past the sensors, or alternatively, the speed of the glider through the ocean water column. The mechanics of glider flight is therefore examined by extending previous flight models to account for the effects of ocean surface waves. It is found that due to the relatively small buoyancy changes used to drive gliders, the surface wave-induced motion, superimposed onto the steady-state motion, follows to a good approximation the motion of the wave orbitals. Errors expected in measuring $\epsilon$ at the ocean near-surface due to wave-induced relative velocities are generally less than $10 \%$. However, pressure perturbations associated with the wave motion can cause significant perturbations in the glider-measured pressure signal and consequently also in the measured vertical glider velocity signal. This effect of surface waves is only present in the shallow water regime. It arises from an incomplete cancellation of the wave-induced pressure perturbation with the hydrostatic component due to vertical glider displacements, whereas for deep-water waves this cancellation is complete.
\end{abstract}

KEYWORDS: In situ oceanic observations; Instrumentation/sensors; Profilers, oceanic

\section{Introduction}

Autonomous, buoyancy-propelled underwater gliders have become an important component of modern ocean observation systems (Testor et al. 2019). An advantage offered by the glider is their versatility in allowing for the combination of many different sensors on a single autonomous platform. One such recent use of gliders is in the measurement of ocean turbulent dissipation rates, and the resulting ocean mixing (Fer et al. 2014; Palmer et al. 2015; Schultze et al. 2017). Turbulence plays a key role in the transport of momentum, matter, and energy in the global oceans and coastal seas. However, in contrast to more standard ocean parameters such as temperature, salinity, and oxygen, the measurement of parameters that characterize ocean turbulence is relatively difficult. Consequently, datasets that contain directly measured parameters quantifying turbulence are relatively scarce (Waterhouse et al. 2014).

A primary reason for this scarcity is because the traditional method to measure turbulence profiles, i.e., the dissipation rate of turbulent kinetic energy ( $\epsilon$, in $\left.\mathrm{W} \mathrm{kg}^{-1}\right)$ with depth, is to use shear probes on a free-falling profiler dropped from a floating platform (usually a ship). This procedure, however, is labor intensive, and has a number of other drawbacks such as being confined to relatively calm weather, and involving uncertainties in the near-surface region due to possible wake effects of the floating platform. Using gliders as host platform, the labor cost can be significantly reduced, and measurements

¿ Denotes content that is immediately available upon publication as open access.

Corresponding author: L. M. Merckelbach, lucas.merckelbach@ hzg.de made possible in both stormy weather (Schultze et al. 2020; Carpenter et al. 2020), as well as in the undisturbed nearsurface ocean (Wijesekera et al. 2020). The use of gliders as an observation platform have thus expanded our sampling capabilities of ocean turbulence, and contributed to a reduction in the scarcity of ocean turbulence measurements.

Despite these advantages in using gliders to measure ocean turbulence, the method is not without its challenges. The computation of $\epsilon$ from velocity shear probes can be shown to be proportional to the water velocity incident on the sensors to the fourth power (Merckelbach et al. 2019). When the glider is used as host platform, it is the glider speed through water $U$ that enters the equation for $\epsilon \propto U^{-4}$. Since gliders are normally not equipped with devices to measure $U$ directly, some type of glider flight model is needed to infer the incident water velocity [e.g., see Fer et al. (2014), St. Laurent and Merrifield (2017), Schultze et al. (2017), and Carpenter et al. (2020) for different examples in the use of such models]. Recently Merckelbach et al. (2019) derived a dynamic glider flight model and assessed the accuracy of this model for estimating the incident water velocity by comparing with the data from velocity sensors added to the glider. A key assumption made in the development of the model is that the glider flies in an ocean water column at rest. This assumption becomes problematic, however, near the ocean surface in the presence of surface gravity waves.

In the present paper, we extent the dynamic flight model of Merckelbach et al. (2019) by considering the effects of surface gravity waves on the motion of the glider itself, on the relative motion of water past the glider induced by these waves, and on

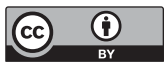

This article is licensed under a Creative Commons Attribution 4.0 license (http://creativecommons.org/ licenses/by/4.0/). 
estimates of the glider vertical velocity. We use linear wave theory to model the interaction between surface waves and a moving submersed body (i.e., the glider), and quantify the extent to which the presence of surface waves leads to additional errors in the estimation of the dissipation rate of turbulent kinetic energy when using dynamic glider flight models. This allows for an understanding, and recommendations, for using gliders to study ocean turbulence at the near-surface (as in, e.g., diurnal warm and rain layers), as well as in shallow coastal seas.

\section{Relative glider velocities induced by surface waves}

Ocean surface gravity waves propagate on the air-water interface causing an oscillation in elevation of the free surface. Below the surface, water parcels in such a wave field follow (almost closed) orbital trajectories. The amplitudes of displacement and velocity decrease with depth, and furthermore depend on the wave amplitude, the wave period, and the water depth. When sufficiently deep in the water column compared to the wavelength of the surface wave, the orbital motions are negligible, and the presence of surface waves is not expected to affect the flight of the glider. This changes, however, when the glider approaches the free surface, and enters a medium whose motion increasingly becomes affected by the orbital motions of the surface waves.

To model this process, we now consider a body of water with zero mean flow, excited by a train of monochromatic linear surface waves. For a neutrally buoyant submersed body, with a length scale much smaller than the wavelength, it is expected that the relative velocities vanish and the body follows the orbital motion exactly. Under normal flight conditions, however, a glider attains a nonzero buoyancy, corresponding to a volume change of 250 and $440 \mathrm{~mL}$, for a standard and an extended buoyancy pump, respectively. Taken relative to the glider volume, which is typically on the order of $50 \mathrm{~L}$, this amounts to about $0.5 \%$ and $1 \%$, respectively. Thus, the departure from a perfectly neutrally buoyant body is small, and therefore, we shall see that relative motions between the water and the glider due to the wave orbitals are also small.

\section{a. Linear wave theory}

It is helpful to first summarize some key results from linear wave theory, the derivation of which can be found in standard texts (e.g., Dingemans 1997; Kundu et al. 2004). We consider a monochromatic surface wave of the form

$$
\zeta=a \cos (k x-\omega t)
$$

where $t$ is time, $\zeta$ the surface elevation, $a$ the wave amplitude, $k$ the wavenumber, and $\omega$ the radial frequency with $\omega=2 \pi T^{-1}$, where $T$ is the wave period. The $x$ and $z$ coordinates represent the horizontal and vertical directions, respectively, with the wave traveling in the positive $x$ direction, and $z$ directed upward. The linearized problem for an even bottom at depth $z=-h$, is given by the velocity potential

$$
\Phi=a \frac{\omega}{k} \frac{\cosh [k(z+h)]}{\sinh (k h)} \sin (k x-\omega t) .
$$

The orbital velocity components in the $x$ and $z$ directions, are denoted by $u_{x}$ and $u_{z}$, and can be found by differentiation of the velocity potential with respect to $x$ and $z$, respectively. The oscillating pressure perturbation produced by the wave motion $\tilde{p}$ follows from the linear dispersion relation, $\omega^{2}=g k \tanh (k h)$, and Bernoulli's equation,

$$
\partial \Phi / \partial t+\tilde{p} / \rho=0
$$

where $g$ is the acceleration due to gravity, and $\rho$ is the density of water, which is assumed to be constant. This yields the set of equations

$$
\begin{aligned}
& u_{x}=a \omega \frac{\cosh [k(z+h)]}{\sinh (k h)} \cos (k x-\omega t), \\
& u_{z}=a \omega \frac{\sinh [k(z+h)]}{\sinh (k h)} \sin (k x-\omega t), \\
& \tilde{p}=\rho g a \frac{\cosh [k(z+h)]}{\cosh (k h)} \cos (k x-\omega t),
\end{aligned}
$$

which will be useful in our analysis that follows.

\section{b. Wave-forced glider flight modeling}

Returning to the dynamics of the glider, we can write Newton's law in an inertial frame of reference for a submerged body as

$$
m_{\mathrm{g}} \dot{\mathbf{V}}=\mathbf{F}+\mathbf{F}_{a}
$$

where the dot denotes differentiation with respect to time, $m_{\mathrm{g}}$ the glider mass, $\mathbf{V}$ the glider velocity (relative to the seabed), $\mathbf{F}$ the external force vector (composed of drag, lift, and buoyancy forces), and $\mathbf{F}_{a}$ a force vector resulting from the acceleration of the water around the body. One reason for an nonzero $\mathbf{F}_{a}$ to arise is when water around the body accelerates in response to an accelerating submerged body in a flow, that, in the absence of the body, would be steady. This effect can be accounted for by an added mass matrix (Newman 1977), and has been included in the dynamic glider flight model by Merckelbach et al. (2019). Another, complementary situation that gives rise to a nonzero vector $\mathbf{F}_{a}$, is when the motion of the body is steady, while the flow (in the absence of the body) is unsteady. Such is the case for an ocean surface wave field, since the wave orbitals result in an accelerating flow in the water column below. The presence of the glider in such a flow causes a disturbance that contributes to $\mathbf{F}_{a}$. Newman (1977, paragraph 4.17) treats this problem in detail for the one-dimensional case, where he makes "the fundamental assumption ... that the non-uniformity of the flow is slowly varying, relative to the length scale of the body." In the present context, this translates to an assumption that the wavelength is much longer than the length of the glider.

To extend the result obtained by Newman (1977) to two dimensions, it is convenient to adopt a reference frame $(\xi, \eta)$, where the $\xi$ axis points along the principal axis toward the nose of the glider, and the $\eta$ axis points upward, perpendicular to the principal axis (Fig. 1). Newman's solution for $\mathbf{F}_{a}$ can then be written as 


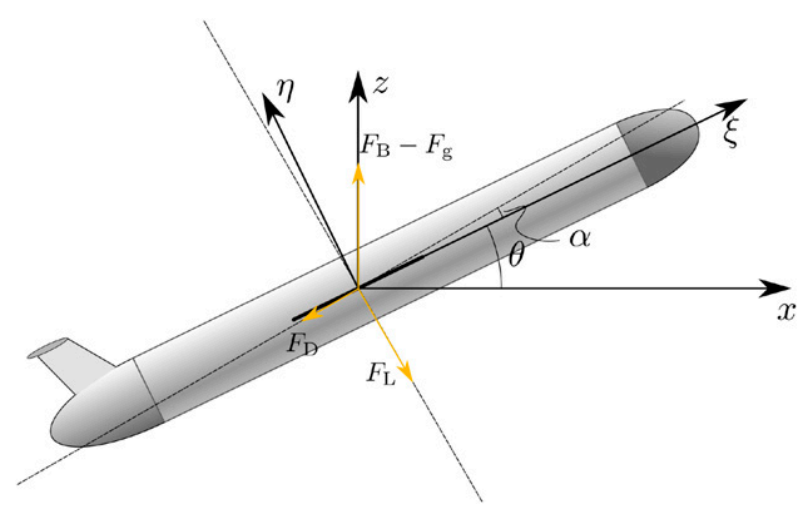

FIG. 1. Schematic diagram of the glider with the relevant coordinate systems, forces, and angles.

$$
\mathbf{F}_{a}=-\left[\begin{array}{cc}
m_{11} & 0 \\
0 & m_{22}
\end{array}\right]\left[\begin{array}{c}
\dot{V}_{\xi} \\
\dot{V}_{\eta}
\end{array}\right]-\left(\forall_{\mathrm{g}} \mathbf{I}+\frac{1}{\rho}\left[\begin{array}{cc}
m_{11} & 0 \\
0 & m_{22}
\end{array}\right]\right)\left[\begin{array}{l}
\frac{\partial \tilde{p}}{\partial \xi} \\
\frac{\partial \tilde{p}}{\partial \eta}
\end{array}\right],
$$

where $\forall_{\mathrm{g}}$ is the glider volume, and $m_{11}$ and $m_{22}$ the added mass coefficients (see also Merckelbach et al. 2019). In this expression the first term on the right-hand side of (6) represents the effect due to an accelerating glider, and has the appearance of a force due to an added mass. The second term on the right-hand side of (6), which is not considered by Merckelbach et al. (2019), represents the effects of the accelerating fluid. This force is sometimes referred to as due to "effective volume."

Decomposing the absolute velocity $\mathbf{V}$ into a water velocity due to wave motion $\mathbf{u}$ and a relative glider velocity through the water $\mathbf{v}$, we can substitute (6) into (5). From (3) it follows that $\dot{\mathbf{u}}=-\rho^{-1} \nabla \tilde{p}$, so that we obtain

$$
\mathbf{M}\left[\begin{array}{c}
\dot{v}_{\xi} \\
\dot{v}_{\eta}
\end{array}\right]=\mathbf{F}-\delta_{V}\left[\begin{array}{c}
\frac{\partial \tilde{p}}{\partial \xi} \\
\frac{\partial \tilde{p}}{\partial \eta}
\end{array}\right]
$$

Herein, the volume anomaly $\delta_{V}$ is given by $\delta_{V}=\forall_{\mathrm{g}}-m_{\mathrm{g}} / \rho$, and the (added) mass matrix $\mathbf{M}$ by

$$
\mathbf{M}=m_{\mathrm{g}} \mathbf{I}+\left[\begin{array}{cc}
m_{11} & 0 \\
0 & m_{22}
\end{array}\right]
$$

Merckelbach et al. (2019) give the external forces vector $\mathbf{F}$ for an $(x, z)$ frame of reference as

$$
\mathbf{F}=\left[\begin{array}{c}
\sin (\theta+\alpha) F_{\mathrm{L}}-\cos (\theta+\alpha) F_{\mathrm{D}} \\
F_{\mathrm{B}}-F_{\mathrm{g}}-\cos (\theta+\alpha) F_{\mathrm{L}}-\sin (\theta+\alpha) F_{\mathrm{D}}
\end{array}\right],
$$

where the pitch angle $\theta$ and the angle of attack $\alpha$ are defined in Fig. 1 . The forces due to buoyancy $F_{\mathrm{B}}$, gravity $F_{\mathrm{g}}$, lift $F_{\mathrm{L}}$, and drag $F_{\mathrm{D}}$, are (Merckelbach et al. 2010, 2019)

$$
F_{\mathrm{g}}=m_{\mathrm{g}} g
$$

$$
\begin{aligned}
F_{\mathrm{B}} & =g \rho\left\{\forall_{\mathrm{g}}\left[1-\epsilon_{\mathrm{c}} P+\alpha_{T}\left(\Theta-\Theta_{0}\right)\right]+\Delta \forall_{\mathrm{bp}}\right\}, \\
F_{\mathrm{D}} & =\frac{1}{2} \rho S U^{2}\left(C_{\mathrm{D}_{0}}+\alpha^{2} C_{\mathrm{D}_{1}}\right), \quad \text { and } \\
F_{\mathrm{L}} & =\frac{1}{2} \rho S U^{2} C_{\mathrm{L}}(\alpha), \quad \text { where } \\
C_{\mathrm{L}}(\alpha) & =A \alpha
\end{aligned}
$$

where $\epsilon_{\mathrm{c}}$ is the coefficient of compressibility, $P$ the water pressure, $\alpha_{T}$, the thermal expansion coefficient of the glider, $\Theta$ the water temperature, $\Theta_{0}$ a reference temperature, $\Delta \forall_{\mathrm{bp}}$ the volume change achieved by the buoyancy engine, $S$ the total surface area of the wings, $U \equiv|\mathbf{v}|$ the magnitude of the glider speed through water, and $C_{\mathrm{D}_{0}}$ and $C_{\mathrm{D}_{1}}$ the parasite and induced drag coefficients, respectively. For small angles of attack $\alpha$, the lift coefficient $C_{\mathrm{L}}$ is assumed to be linear in $\alpha$, with proportionality coefficient $A$, the lift angle coefficient. ${ }^{1}$

At this stage, Eq. (7) presents a complete model for the relative glider velocity $\mathbf{v}$. To solve this equation for $\mathbf{v}$, we perturb it relative to the steady-state solution. We write $\mathbf{v}=$ $\mathbf{v}_{0}+\mathbf{v}_{1}$, and $\mathbf{F}=\mathbf{F}_{0}+\mathbf{F}_{1}$, where subscripts 0 and 1 denote the steady-state solution and the oscillating component due to the waves, respectively. Substituting the perturbations into (7), we can separate the result into a steady-state part and an oscillating part, respectively, as

$$
\begin{gathered}
0=\mathbf{F}_{0}, \\
\mathbf{M}\left[\begin{array}{c}
\dot{v}_{\xi 1} \\
\dot{v}_{\eta 1}
\end{array}\right]=\mathbf{F}_{1}-\delta_{V}\left[\begin{array}{l}
\frac{\partial \tilde{p}}{\partial \xi} \\
\frac{\partial \tilde{p}}{\partial \eta}
\end{array}\right] .
\end{gathered}
$$

As per (10), the force vector $\mathbf{F}$ depends on $\mathbf{v}$ through $U^{2}$ and $\alpha$. The steady-state parameters can be represented in an $(x, z)$ frame of reference as

$$
U_{0}^{2}=v_{x 0}^{2}+v_{z 0}^{2} \quad \text { and } \quad \tan \left(\theta+\alpha_{0}\right)=\frac{v_{z 0}}{v_{x 0}},
$$

which allows $\mathbf{F}_{0}$ to be expressed in $\mathbf{v}_{0}$. Consequently, $v_{x 0}$ and $v_{z 0}$ can be determined from (11a).

For the exact parameters $U$ and $\alpha$, we can derive the linearized approximations (see appendix for details)

$$
\begin{gathered}
U^{2}=U_{0}^{2}+2\left(v_{x 0} v_{x 1}+v_{z 0} v_{z 1}\right), \\
\alpha=\alpha_{0}+\frac{v_{x 0} v_{z 1}-v_{z 0} v_{z 1}}{U_{0}^{2}},
\end{gathered}
$$

where it has been assumed that the oscillating part of the velocity is much smaller than the steady-state velocity, a fact that will later be verified. Substituting these expressions into (9) and subtracting $\mathbf{F}_{0}$ from the result, we obtain

\footnotetext{
${ }^{1}$ In Merckelbach et al. (2019) the lift angle coefficient is denoted by $a$. In this work the symbol $a$ is reserved for the wave amplitude.
} 


$$
\left[\begin{array}{l}
F_{x 1} \\
F_{z 1}
\end{array}\right]=\frac{1}{2} \rho S \mathbf{f}\left[\begin{array}{l}
v_{x 1} \\
v_{z 1}
\end{array}\right] .
$$

Herein, the elements of $\mathbf{f}$ are given by

$$
\begin{aligned}
f_{11}= & +\left[2 A \alpha_{0} v_{x 0}-\left(C_{D}+A\right) v_{z 0}\right] \sin \left(\theta+\alpha_{0}\right) \\
& -\left(A \alpha_{0} v_{z 0}-2 C_{D} v_{x 0}\right) \cos \left(\theta+\alpha_{0}\right), \\
f_{12}= & +\left[2 A \alpha_{0} v_{z 0}+\left(C_{D}+A\right) v_{x 0}\right] \sin \left(\theta+\alpha_{0}\right) \\
& +\left(A \alpha_{0} v_{x 0}-2 C_{D} v_{z 0}\right) \cos \left(\theta+\alpha_{0}\right), \\
f_{21}= & -\left[2 A \alpha_{0} v_{x 0}-\left(C_{D}+A\right) v_{z 0}\right] \cos \left(\theta+\alpha_{0}\right) \\
& -\left(A \alpha_{0} v_{z 0}+2 C_{D} v_{x 0}\right) \sin \left(\theta+\alpha_{0}\right), \\
f_{22}= & -\left[2 A \alpha_{0} v_{z 0}+\left(C_{D}+A\right) v_{x 0}\right] \cos \left(\theta+\alpha_{0}\right) \\
& +\left(A \alpha_{0} v_{x 0}+2 C_{D} v_{z 0}\right) \sin \left(\theta+\alpha_{0}\right) .
\end{aligned}
$$

Expressed in $(\xi, \eta)$ coordinates, $(15)$ reads

$$
\left[\begin{array}{l}
F_{\xi 1} \\
F_{\eta 1}
\end{array}\right]=\frac{1}{2} \rho S \mathbf{T}^{\mathrm{T}} \mathbf{f} \mathbf{T}\left[\begin{array}{l}
v_{\xi 1} \\
v_{\eta 1}
\end{array}\right],
$$

where the transformation matrix $\mathbf{T}$ is given by

$$
\mathbf{T}=\left[\begin{array}{cc}
\cos (\theta) & -\sin (\theta) \\
\sin (\theta) & \cos (\theta)
\end{array}\right]
$$

With (20), (11b) can be written as

$$
\left[\begin{array}{c}
\dot{v}_{\xi 1} \\
\dot{v}_{\eta 1}
\end{array}\right]-\mathbf{B}\left[\begin{array}{c}
v_{\xi 1} \\
v_{\eta 1}
\end{array}\right]=\mathbf{g}(t)
$$

in which

$$
\mathbf{B}=(1 / 2) \rho S \mathbf{M}^{-1} \mathbf{T}^{\mathrm{T}} \mathbf{f} \mathbf{T}
$$

and

$$
\mathbf{g}(t)=-\delta_{V} \mathbf{M}^{-1} \mathbf{T}^{\mathrm{T}}\left[\begin{array}{l}
\partial_{x} \tilde{p} \\
\partial_{z} \tilde{p}
\end{array}\right] .
$$

Equation (22) represents a nonhomogeneous first-order system of ordinary differential equations describing the flight of the glider (in glider-centered coordinates) relative to the steady-state solution (i.e., the flight that occurs in the absence of any perturbations due to surface waves). The $\boldsymbol{g}(t)$ vector on the right hand side describes the forcing of the system by the surface wave motion through its dependence on $\tilde{p}$, and the (steady state) flight characteristics of the system are felt through the matrix B. Specific solutions to this equation are now examined for typical oceanographic conditions.

\section{c. Application to glider flight in surface waves}

The natural response of the glider due to an external disturbance can be investigated by solving the eigenvalue problem for the homogeneous form of (22), that is, for $\mathbf{g}(t)=0$. In this case, we find two negative eigenvalues, indicating that the glider dynamic behavior returns to the steady-state conditions after a small perturbation. The longest response time, computed from the reciprocal of the smallest eigenvalue, is about $13 \mathrm{~s}$ for nominal flight conditions. The forcing term (24) introduces two more time scales: the wave period and a time scale $k\left|v_{z}\right|$, that arises from the vertical motion of the glider $\left(v_{z}\right)$ and the length scale of the vertical structure of the wave field. For the wave periods considered herein, the latter time scale, however, is long compared to the wave period, so that it is permissible to simplify the mathematical problem by regarding the depth dependency to be quasi constant in time. This means that the spatial gradients in the pressure anomalies in (24) can be evaluated for constant $z$ during a wave period.

To find a solution to the forced system (22), we rewrite the forcing vector $\mathbf{g}(t)$ as

$$
\mathbf{g}(t)=\mathbf{G}\left[\begin{array}{l}
\sin (k x-\omega t) \\
\cos (k x-\omega t)
\end{array}\right]=\mathbf{g}_{0} \sin (k x-\omega t)+\mathbf{g}_{1} \cos (k x-\omega t),
$$

where

$$
\begin{aligned}
\mathbf{G}= & {\left[\begin{array}{cc}
\mid & \mid \\
\mathbf{g}_{0} & \mathbf{g}_{1} \\
||
\end{array}\right]=-\delta_{V} \frac{a g k \rho}{\cosh (k h)} \mathbf{M}^{-1} \mathbf{T}^{\mathrm{T}} } \\
& \times\left[\begin{array}{cc}
-\cosh [k(h+z)] & 0 \\
0 & \sinh [k(h+z)]
\end{array}\right] .
\end{aligned}
$$

Then we use the ansatz

$$
\mathbf{v}_{1}=\mathbf{p}_{0} \sin (k x-\omega t)+\mathbf{p}_{1} \cos (k x-\omega t),
$$

and after substitution into (22) we obtain

$$
\begin{aligned}
& \mathbf{p}_{0}=-\left(\mathbf{B}^{2}+\omega^{2} \mathbf{I}\right)^{-1}\left(\omega \mathbf{g}_{1}+\mathbf{B} \mathbf{g}_{0}\right), \\
& \mathbf{p}_{1}=+\left(\mathbf{B}^{2}+\omega^{2} \mathbf{I}\right)^{-1}\left(\omega \mathbf{g}_{0}-\mathbf{B g}_{1}\right) .
\end{aligned}
$$

Due to the complexity of the expressions involved, there is not much insight to be gained by elaborating the result for $\mathbf{v}_{1}$ further. We can, however, deduce from (26) that the amplitude of the relative velocity perturbation is proportional to the wave amplitude, and it exhibits a depth dependency, which, in the deep-water wave limit, is given by the factor $e^{k z}$. The behavior of the solution is explored graphically in Fig. 2, with results shown for waves for a range of wave periods, and an amplitude of $1 \mathrm{~m}$ in water depths of 40 and $1000 \mathrm{~m}$. Furthermore, nominal flight conditions are assumed, for which the relevant parameter settings are listed in Table 1. Figure 2 shows the amplitude of the perturbed velocity in the $\xi$ direction (Fig. 2a), and $\eta$ direction (Fig. 2b) as a function of depth for a number of different wave periods. In general, we find that the amplitude of $v_{\xi 1}$ is larger than that of $v_{\eta 1}$, and that the amplitudes of both components are largest near the water surface, where also shorter wave periods result in larger relative velocity perturbations. The vertical structure of the solution is shaped by the linear combination of the horizontal and vertical pressure gradients, so that a rapid decay in amplitude is observed with 

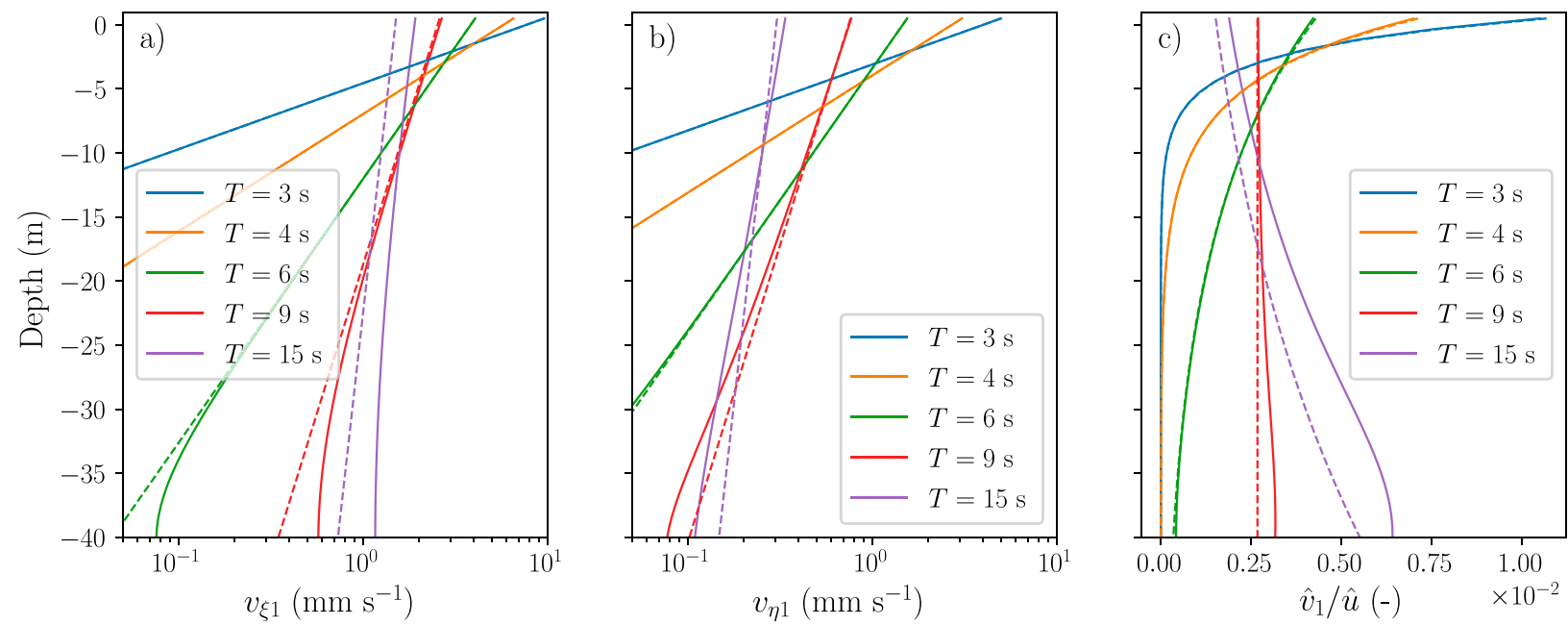

FIG. 2. The amplitude of the perturbed velocity in (a) $\xi$ direction and (b) $\eta$ direction, as function of depth for water depths of $40 \mathrm{~m}$ (solid lines) and $1000 \mathrm{~m}$ (dashed lines), for a range of wave periods. The wave amplitude is set to $1 \mathrm{~m}$, whereas nominal flight parameters values are assumed (pitch angle of $25^{\circ}$ and a variable displacement achieved by the buoyancy pump of $250 \mathrm{~mL}$ ). Further flight parameter settings are listed in Table 1. (c) The ratio of the amplitudes of the relative velocity perturbation and the wave orbital velocity.

depth, but less so for larger wave periods and shallower water depths, facts that follow from linear wave theory in (4).

The ratios of the amplitudes of the relative velocity perturbation $\mathbf{v}_{1}$ and the wave orbital velocity $\mathbf{u}$ are shown in Fig. $2 c$. The key deduction that can be made from this panel is that the magnitude of the wave-induced relative velocity of the glider is less than $1 \%$ of the orbital velocity magnitude. This means that the glider position is significantly influenced by the orbital motion of the waves, and the glider's perturbed motion due to the surface waves follows, to a good approximation, the wave orbitals. We conclude that because the nominal glider speed through water is significantly larger than the relative velocity perturbations, the glider dynamics problem can be treated as if waves were absent. The implications of this small relative velocity perturbation for the estimation of the dissipation rate of turbulent kinetic energy as measured from microstructure sensor data, are further discussed in section 4 a.

\section{Surface wave effects in the estimation of vertical velocity}

In the application of glider flight models to estimate the turbulent dissipation rate from microstructure sensors, a number of studies have utilized the vertical velocity of the glider as an input to the flight model (Schultze et al. 2017; St. Laurent and Merrifield 2017; Scheifele et al. 2018; Moldotsov et al. 2020). The determination of vertical glider velocities is arrived at through differentiation of the glider pressure measurement. In this section we show that alterations in the pressure measurement can arise from the presence of surface waves, and that these can affect the calculation of vertical glider velocity.

Equation (4c) suggests that a surface wave induces a pressure perturbation that may affect the pressure reading by the glider pressure sensor. To investigate to what extent wave induced pressure perturbations are a concern, we write the instantaneous pressure, observed by a glider pressure sensor, as

$$
p=\int_{z_{\mathrm{g}}}^{0} \rho g d z^{\prime}+\tilde{p}
$$

where $z_{\mathrm{g}}(t)$ is the vertical position of the glider relative to the still water level, and, for the sake of simplicity, any variability in the in situ density is ignored. The vertical position of the glider can be decomposed into

$$
z_{\mathrm{g}}=z_{0}+\left.\tilde{z}\right|_{z=z_{0}}
$$

where $\tilde{z}$ is the vertical displacement of the glider, relative to $z_{0}$, due to the wave motion, giving

$$
p=-\rho g\left(z_{0}+\tilde{z}\right)+\tilde{p} .
$$

Note that the derivation of the potential $\Phi$ uses a linearization of the surface boundary condition, effectively ignoring vertical displacements of the water surface. To remain consistent with the derivation of $\Phi$, and therefore with the expression for $\tilde{p}$, we have integrated (30) to the upper integration limit $z^{\prime}=0$, rather than to the instantaneous water surface elevation $z^{\prime}=\zeta$. This can readily be verified if we set $z_{0}=0$ in (31).

Analogous to the excursion of a water parcel following a wave orbital, $\tilde{z}$ follows from integration of (4b) with respect to time, evaluated at $z_{0}=z_{0}$, yielding

TABLE 1. Flight model parameter settings.

\begin{tabular}{lcc}
\hline \hline$\theta=25^{\circ}$ & $m_{\mathrm{g}}=55 \mathrm{~kg}$ & $m_{11}=0.2 m_{\mathrm{g} \mathrm{kg}}$ \\
$\delta_{V}=250 \times 10^{-6} \mathrm{~m}^{-3}$ & $\forall=55 \times 10^{-3} \mathrm{~m}^{-3}$ & $m_{22}=0.9 m_{\mathrm{g} \mathrm{kg}}$ \\
$\rho=1000 \mathrm{~kg} \mathrm{~m}^{-3}$ & & \\
\hline
\end{tabular}



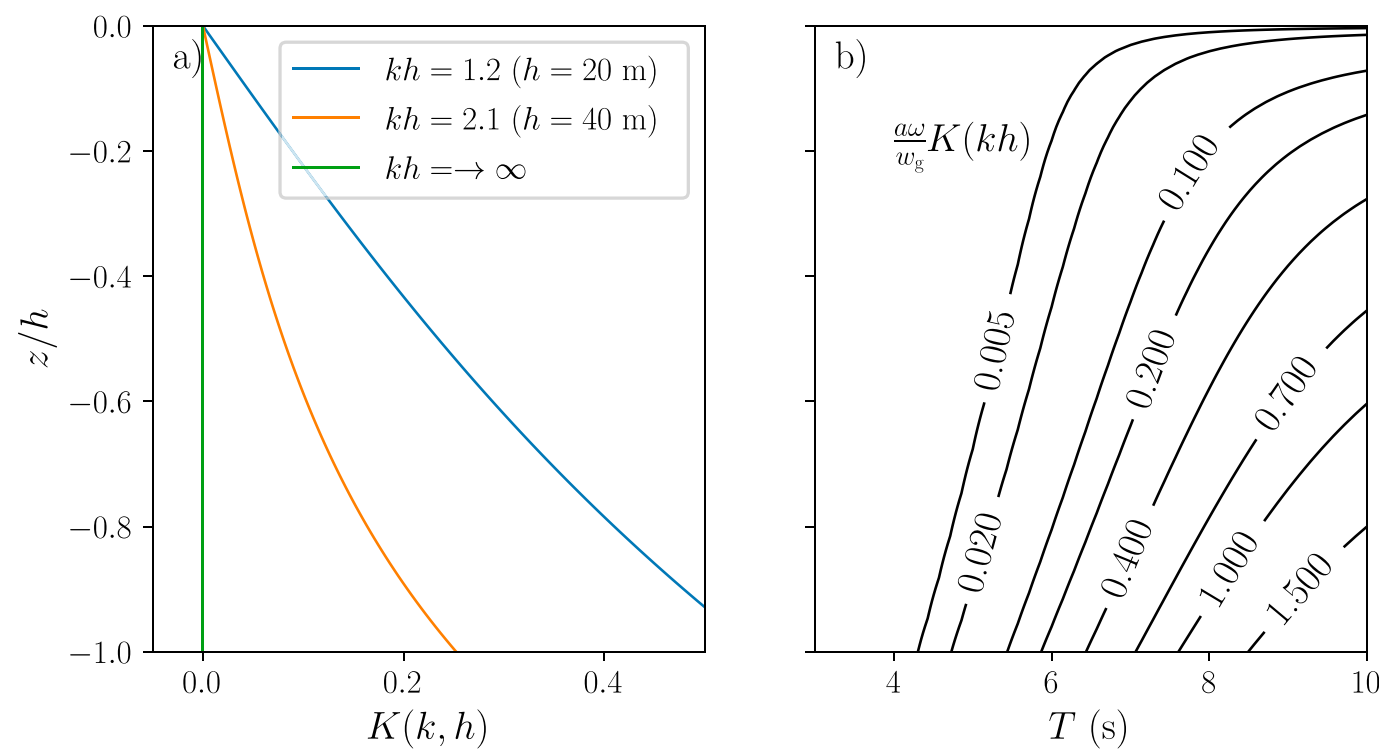

FIG. 3. (a) The behavior of $K=\cosh \left[k\left(h+z_{0}\right)\right] / \cosh (k h)-\sinh \left[k\left(h+z_{0}\right)\right] / \sinh (k h)$ as function of the relative depth $z / h$ for values of $k h=\{1.2,2.1, \infty\}$. The depths correspond to a monochromatic wave with a wave period of $9 \mathrm{~s}$. (b) Wave-induced error in the nondimensional vertical glider velocity observation, as function of depth and wave period for $a=1 \mathrm{~m}, h=40 \mathrm{~m}$, and $w_{\mathrm{g}}=10 \mathrm{~cm} \mathrm{~s}^{-1}$.

$$
\tilde{z}=a \frac{\sinh \left[k\left(h+z_{0}\right)\right]}{\sinh (k h)} \cos (k x-\omega t) .
$$

Combining (4c), (32) and (33), we can write the observed pressure as

$$
p=-\rho g z_{0}+\rho g a K \cos (k x-\omega t),
$$

where we define

$$
K=\frac{\cosh \left[k\left(h+z_{0}\right)\right]}{\cosh (k h)}-\frac{\sinh \left[k\left(h+z_{0}\right)\right]}{\sinh (k h)} .
$$

This expression shows that the observed pressure consists of the hydrostatic pressure, augmented with a wavedependent term. The wave-dependent term accounts for the wave-pressure anomaly (first term in $K$ ) and the pressure anomaly due to the vertical orbital excursion (second term in $K$ ). For circular orbitals in the deep water limit, both pressure contributions counteract each other and cancel. Indeed, for circular orbitals at $k h \gg 1$, it can be readily shown that $K \rightarrow 0$. However, when waves enter shallow water, the orbital motion is forced to become more horizontal, rendering the orbitals elliptic. While the pressure anomaly remains unaffected, the vertical excursion length is reduced, and both effects become unbalanced, resulting in $K>0$.

Figure 3 depicts the dependency of $K$ on depth and $k h$. For deep-water waves, $K=0$ for all depth, as argued above. For intermediate and shallow water waves, $K$ is larger than zero and increases with depth. The glider depth that would be inferred from dividing the observed pressure by $-\rho g$, as is common oceanographic procedure, is given by

$$
z_{p}=z_{0}-a K \cos (k x-\omega t) .
$$

This apparent glider depth is therefore equal to the undisturbed depth $z_{0}$ augmented with a modulated surface elevation signal. This signal vanishes for deep-water waves, but increases with depth for shallow and intermediate water waves (Fig. 3).

A common use of the glider depth measurement is to map sensor parameters, such as temperature and salinity, onto a vertical coordinate. Since the time-averaged vertical coordinate of a water parcel that follows a wave orbital, is equal to $z_{0}$, this is also the preferred vertical coordinate to use in mapping water properties. Therefore, we consider the wave-dependent pressure (depth) anomaly to be a pressure (depth) error. To illustrate this error, Fig. 3 a shows the specific example of a wave with $9 \mathrm{~s}$ period. In this case, near-bed depth errors can be as large as $30 \%$ of the surface wave amplitude in $40 \mathrm{~m}$ water depth, increasing to even $50 \%$ in water depths of $20 \mathrm{~m}$; a potentially significant fraction of the total depth, depending on wave amplitudes.

The error in the vertical position also propagates through to the observed vertical glider velocity estimate, which can be computed based on the glider pressure measurement as $w_{\mathrm{p}}=-(\rho g)^{-1} d p / d t$. By differentiation of (34) we find

$$
w_{\mathrm{p}} \approx \frac{d z_{0}}{d t}-a \omega K \sin (k x-\omega t),
$$

where we have neglected terms that are on the order of the glider speed to the wave phase speed. With a nominal glider speed of $U=0.4 \mathrm{~m} \mathrm{~s}^{-1}$ these terms can safely be neglected for waves that are long compared to the length of the glider. Thus, the observed vertical glider velocity contains an oscillating error term with an amplitude of $a \omega K$. As an example, consider a coastal sea with a water depth of $40 \mathrm{~m}$, and a wave 

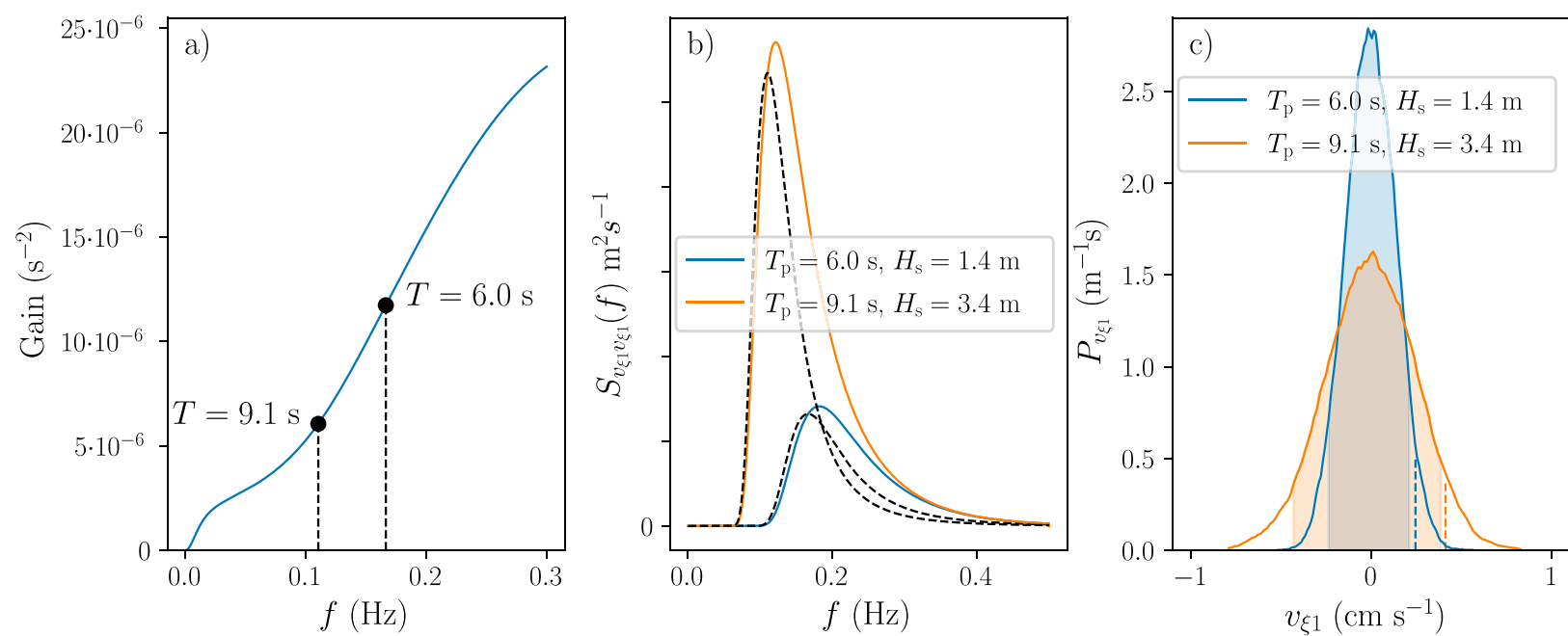

FIG. 4. (a) Gain of the $v_{\xi 1}$ signal as function of frequency $f$. (b) $v_{\xi 1}$ spectra (solid lines) for the two wave conditions listed in the legends, assuming a PM-wave spectrum. The dashed lines show the corresponding scaled PM spectra. (c) Estimated probability density functions of the instantaneous velocity $v_{\xi 1}$ for the two wave conditions. The shaded regions delimit the velocity ranges comprising of $90 \%$ of the observations, whereas the dashed vertical lines denote the $\hat{v}_{\xi 1}$ resulting from surface waves with $T=T_{\mathrm{p}}$ and $H=H_{\mathrm{s}}$.

amplitude of $1 \mathrm{~m}$. The amplitude of the wave dependent term in the observed vertical velocity, relative to a nominal vertical glider speed of $w_{\mathrm{g}}=0.10 \mathrm{~m} \mathrm{~s}^{-1}$, is shown in Fig. 3b. For small wave periods, the relative wave contribution is small. However, under windy conditions when wave periods easily reach $9 \mathrm{~s}$, it can be seen that the wave-induced error can be even larger than the nominal vertical glider velocity.

\section{Discussion}

\section{a. Implications for turbulent dissipation rate measurements}

In section 2, we examined how surface waves can induce an oscillatory flow relative to the glider's steady flight. The analysis presented assumed idealized monochromatic waves of unit amplitude. The amplitudes of the oscillatory relative flow components were shown to be linearly proportional to the wave height and were a maximum near the surface (Fig. 2). We now demonstrate the implications of these findings in a practical context; we examine the extremes in wave-induced relative velocities that a glider is likely to encounter in realistic ocean settings.

Observed and modeled wind-wave data are often condensed into two representative parameters: a peak period $T_{\mathrm{p}}$, where the wave spectrum reaches maximum spectral density, and a significant wave height $H_{\mathrm{s}}$, the mean of the highest third of the observed wave heights (with wave height defined as twice the amplitude $a$ ). Together they serve to define a spectrum of waves that spans a range of frequencies $f($ in $\mathrm{Hz}$ ), and amplitudes. To account for this range, we expand the monochromatic analysis of section 2 to account for a spectrum of frequencies that is characteristic of realistic ocean wave fields. This is done by considering the surface wave field as input, and the wave-induced relative glider velocities as outputs, to a system with a transfer function described by (27). The response of the relative velocities to an oscillating wave forcing-in signal processing normally referred to as gain - can readily be computed, and is shown for the near-surface region (evaluated at $z_{0}=-1 \mathrm{~m}$ ) in Fig. $4 \mathrm{a}$ as a function of $f$. The gain is seen to approach zero in the limit of $f \rightarrow 0$, as expected. For the frequency range where waves still can be considered long compared to the glider length (wave periods $>3 \mathrm{~s}$ ) the gain increases nearly linearly with increasing frequency. Therefore, the shapes of the wave and wave-induced relative velocity spectra are not congruent, rather the wave-induced relative velocity spectrum is biased toward higher frequencies.

In computing the turbulent dissipation rate from microstructure measurements, it is important to quantify the instantaneous velocities $\left(v_{\xi 1}\right)$ a glider may encounter. To gain insight into this, we determine probability density functions for $v_{\xi 1}$, computed from time series constructed for a spectrum of $v_{\xi 1}$ by assuming a random phase model. [Since freak waves and other extreme wave events (e.g., Teutsch et al. 2020) are in this context statistically insignificant, they are not considered].

We illustrate this process here by adopting the empirical Pierson-Moskowitz (PM) energy spectrum for ocean waves (e.g., Tucker 1991), and computing the corresponding spectrum of $v_{\xi 1}$. Figure $4 \mathrm{~b}$ shows the power spectra for $v_{\xi 1}$ at $z_{0}=-1 \mathrm{~m}$, resulting from two PM spectra with $T_{\mathrm{p}}=6$ and $9 \mathrm{~s}$. For reference, the PM wave input spectra, scaled by the power gains for the respective peak frequencies, are indicated by the dashed lines. Although the power spectra for $v_{\xi 1}$ have roughly the same shape as the PM spectra, it is clearly seen that the velocity spectra have a stronger high-frequency content. Hence, the peak frequency and peak value of the wave-induced relative velocity spectrum are about $5 \%$ higher than their wave spectrum equivalents.

The corresponding estimated probability density functions of the instantaneous velocity $v_{\xi 1}$ are shown in Fig. $4 c$. The instantaneous velocities have Gaussian-like distributions with a 
TABLE 2. Site details of used wave data.

\begin{tabular}{ccccccc}
\hline \hline Site & Latitude & Longitude & Period & Depth & Resolution & Source \\
\hline German Bight & $54^{\circ} 40.8^{\prime} \mathrm{N}$ & $6^{\circ} 43.8^{\prime} \mathrm{E}$ & 2019 & $38 \mathrm{~m}$ & $3 \mathrm{~h}$ & Model data \\
Bay of Biscay & $45^{\circ} 12^{\prime} \mathrm{N}$ & $5^{\circ} 00^{\prime} \mathrm{W}$ & 2018 & $4700 \mathrm{~m}$ & $1 \mathrm{~h}$ & Mooring data \\
\hline
\end{tabular}

zero mean. The shaded regions denote the velocities that can be expected to occur in $90 \%$ of the observations in a sea state described by the PM spectra. The vertical dashed lines mark the amplitudes of $v_{\xi 1}$ for monochromatic wave trains with $T=T_{\mathrm{p}}$ and $H=H_{\mathrm{s}}$, and nearly coincide with the edges of the corresponding shaded region. Hence, for a sea state that can be described by a PM spectrum, roughly $95 \%$ of the wave-induced relative velocities $v_{\xi 1}$ the glider encounters are smaller than those computed with a monochromatic wave with the PM spectral parameters $T_{\mathrm{p}}$ and $H_{\mathrm{s}}$, denoted by $\hat{\boldsymbol{v}}_{\xi 1}\left(T_{\mathrm{p}}, H_{\mathrm{s}}\right)$. We write this mathematically as

$$
P\left[v_{\xi 1}<\hat{v}_{\xi 1}\left(T_{\mathrm{p}}, H_{\mathrm{s}}\right)\right] \approx 0.95
$$

To assess the practical implications of this result, we consider the wave conditions typical for two contrasting coastal locations: (i) the German Bight in the North Sea, a shallow shelf sea with a water depth of $\sim 40 \mathrm{~m}$, and (ii) the central deep basin of the Bay of Biscay on the northern coast of Spain, with a water depth of $\sim 4700 \mathrm{~m}$ (see Table 2 for further details). For each site, a year's worth of wave data are used, originating from a wave model for the German Bight (Staneva et al. 2014, 2015) and measurements made using a wave buoy in the Bay of Biscay. The wave data, which are described by the spectral parameters $H_{\mathrm{s}}$ and $T_{\mathrm{p}}$, are processed into probability distributions (as above), and presented in Fig. 5b (German Bight) and Fig. 5c (Bay of Biscay) using shades of red as a measure of the probability density. The PM spectrum is a one-parameter model, taking the wind speed as input, and models a fully developed sea state. A property of the PM spectrum is that there is an assumed relationship between $T_{\mathrm{p}}$ and $H_{\mathrm{s}}$, as shown by the blue lines in Figs. 5b and 5c. This is clearly not the case for the datasets presented, which show a much broader distribution. This discrepancy can be attributed to both the model and the measurement data reflecting a sea state that is not fully developed due to time varying wind conditions and limited fetch. In addition, in shallow water (German Bight) bathymetry may also play a role. A multiparameter JONSWAP-type spectrum (e.g., Tucker 1991) may provide a more accurate description for the spectra of the wave data used here. However, because the simpler PM spectrum has a significantly broader frequency range than the JONSWAP spectrum, the probability density function of the relative vertical velocity, resulting from a JONSWAP spectrum is narrower, and therefore (38) provides a conservative estimate.

The dashed lines in Figs. 5b and 5c roughly delimit the region of observed pairs of peak frequency and significant wave height for the respective datasets, as determined by eye. The solid lines mark the upper 95th percentiles. The amplitudes of the wave-induced relative velocities are computed along the dashed and solid lines, again evaluated near the surface at $z_{0}=-1 \mathrm{~m}$ and presented in Fig. 5a, maintaining the same line
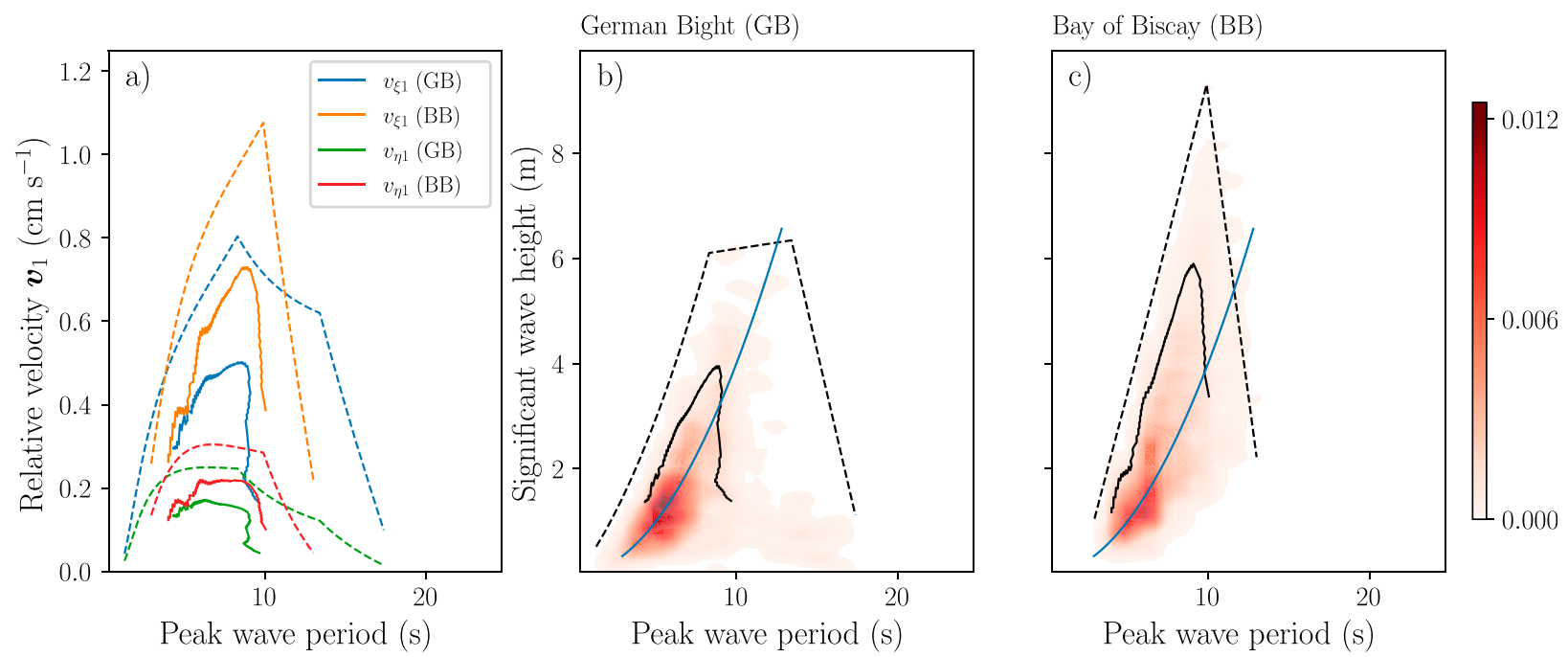

FIG. 5. Near-surface wave-induced relative velocities. (a) Maximum amplitude of relative velocities in $\xi$ and $\eta$ directions for the German Bight (GB) and Bay of Biscay (BB), computed along the solid and dashed lines in the other panels. (b),(c) Estimated probability density maps of the wave climate in the German Bight (shallow water, $40 \mathrm{~m}$ water depth) and Bay of Biscay (deep water, $4700 \mathrm{~m}$ water depth), respectively, where the dashed black lines indicate the extremities of the observations, and the solid black lines (part of) the 90th percentile. 
style convention. We observe that $v_{\eta 1}$ remains below $3 \mathrm{~mm} \mathrm{~s}^{-1}$, even during the most extreme sea states (red and green curves). The component incident to the sensors, $\boldsymbol{v}_{\xi 1}$, remains below 1 and $0.8 \mathrm{~cm} \mathrm{~s}^{-1}$ for the deep and shallow sites, respectively, with the 95th-percentile values dropping further to 7 and $5 \mathrm{~mm} \mathrm{~s}^{-1}$, respectively. Based on these results, we find that a conservative upper bound on the wave-induced along glider velocities in the most extreme sea states is $1 \mathrm{~cm} \mathrm{~s}^{-1}$. This translates into an error in the computation of turbulence dissipation rates, based on the uncertainty in $U$, that remains below $10 \%$ for a typical glider speed of $40 \mathrm{~cm} \mathrm{~s}^{-1}$. This is small compared to the roughly factor-of-2 uncertainty that is commonly cited for dissipation measurements from shear probes (Moum et al. 1995), and is comparable to the errors associated with the glider flight model itself (Merckelbach et al. 2019).

Finally, we note that this analysis neglects the possibility of wave-breaking effects that are certainly present in many sea states that a glider is likely to encounter. This will affect the velocities in the very near surface (i.e., upper couple of meters), and this is a limitation to the analysis presented herein. This limitation should be kept in mind when glider flight in the near surface region is considered.

\section{b. Depth projection of measured parameters}

A glider mission was run in the German Bight of the North Sea during the passage of a summer storm on 10 August 2019. A significant wave height of $4.5 \mathrm{~m}$ and a peak period of $9.3 \mathrm{~s}$ for the glider location $\left(54^{\circ} 40.8^{\prime} \mathrm{N}, 6^{\circ} 43.8^{\prime} \mathrm{E}\right)$ were computed using a preoperational wave model (Staneva et al. 2014), run for the German Bight/southern North Sea region. At this location the water depth is $\sim 34 \mathrm{~m}$, so that waves at this peak period have $k h \approx 1.6$. The near-bed horizontal orbital velocity amplitude decay [i.e., Eq. (4a)] amounts to approximately 30\%, indicating that the influence of waves with $9 \mathrm{~s}$ period reached the sea bed. We therefore expect that near the sea bed, the depth of the glider, computed from the CTD pressure sensor, is at least partially corrupted with a wave-induced signal.

To remove this signal from the inferred depth, we apply a low-pass filter with, in this case, a cutoff frequency of $1 / 15 \mathrm{~Hz}$ to the observed (i.e., apparent) depth position $z_{p}$, as computed directly from the CTD pressure data. This was found to provide a reasonable estimate of the glider vertical position $\hat{z}_{0}$ with all effects of wave contamination removed. Figure 6 shows the results from four consecutive glider profiles from this dataset. To isolate the effects of the waves, the blue curves show the difference of the apparent depth $z_{p}$, to the depth with wave effects removed $\hat{z}_{0}$, as function of $\hat{z}_{0}$. Near the surface the difference is small, but grows with increasing depth, in line with our analysis in (36). The difference envelope calculated from (36) is shown in shaded blue, using a wave period of $9.3 \mathrm{~s}$ and a wave amplitude of $2.3 \mathrm{~m}$ ( $4.5 \mathrm{~m}$ wave height), in accordance with the preoperational wave model. In addition, for these profiles we are able to produce measurements of $z_{\mathrm{g}}$, the glider height above the sea bed. This is done using a glider-mounted, downward-looking Teledyne RDI Explorer $600 \mathrm{kHz}$ Doppler velocity log (DVL), as described in Merckelbach et al. (2019). Subtracting this value of $z_{\mathrm{g}}$ from the water depth $z_{0}$ gives an estimate of the vertical glider excursion due to wave orbital

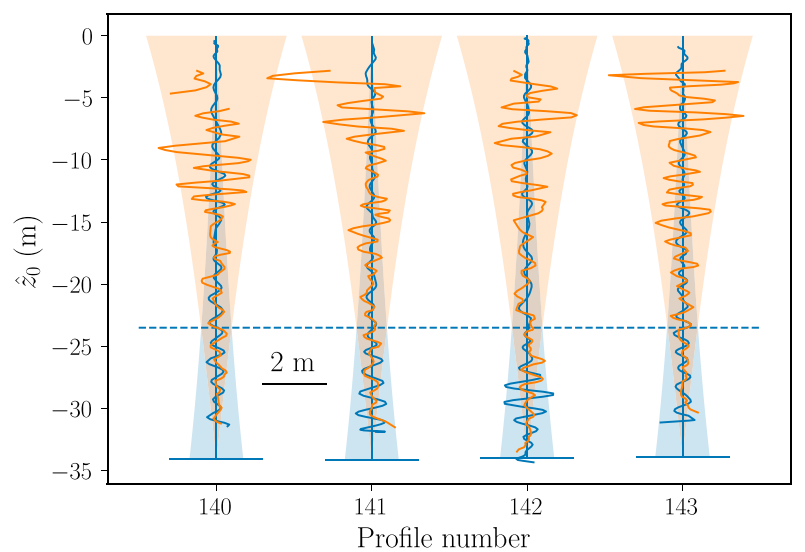

FIG. 6. Depth anomaly using CTD pressure data (blue) and depth anomaly using DVL altitude data (orange) for four consecutive profiles during 2043-2112 UTC 10 Aug 2019. The dashed line indicates the depth where the blue and orange envelopes coincide.

motions, and is shown by the orange curves in Fig. 6. The corresponding amplitude envelope of the vertical orbital excursion length is indicated by the orange shaded region.

The anomalies of both depth estimates appear to be reasonably well delimited by the respective envelopes, bearing in mind that the waves are not monochromatic, but have dominant frequencies spread around a band that is centered on a peak period of $9.3 \mathrm{~s}$. This spread in frequency gives rise to wave groups, the effect of which is apparent in the measured data. The envelopes for the pressure and the altitude based anomalies coincide at a depth of approximately $23.5 \mathrm{~m}$. It is also at this depth that the pressure (blue curves) and altitude (orange curves) anomalies coincide. Furthermore, it is noted that, both anomalies are seen to have the same phase in vertical space, which is consistent with both anomalies having the same phase in time, see Eqs. (33) and (36). The consistency of this analysis suggests that the preferred measure of glider depth is $z_{0}$, which can be estimated by applying a low-pass filter on the pressurederived depth, using a cutoff frequency small enough to remove the wave-induced anomalies. Based on this recommendation, measured parameters, such as temperature and salinity, are projected to a depth coordinate that is not perturbed by the influence of surface waves.

\section{Conclusions}

In this work, we quantified the effects of a surface wave field on the flight characteristics of ocean gliders. Two primary effects were identified: (i) the orbital motion of the surface waves results in a flow past the glider that deviates from the prediction of standard flight models, and (ii) glider vertical positions, and therefore also vertical velocity estimates, can be altered by wave-induced pressure and depth perturbations to the measured pressure signal. These effects were quantified by using a linear surface wave field as a forcing term in a glider flight model that accounts for both added mass and "effective volume" terms, thus extending the Merckelbach et al. (2019) analysis to include surface wave effects. The results show that 
the wave-induced velocities relative to the glider are generally small, due to the small net (positive or negative) buoyancy of the glider. In other words, the glider's perturbed motion follows closely the wave's orbital motion, and experiences little relative motion resulting from the wave forcing. By applying the extended flight model to wave conditions in two coastal study regions, characteristic of a shelf sea and a deep ocean basin, we conclude that the wave-induced relative velocities remain less than $1 \mathrm{~cm} \mathrm{~s}^{-1}$ for the most extreme sea states. When using gliders as a platform for turbulence microstructure observations, the associated error in the dissipation rate of turbulent kinetic energy remains under $10 \%$, and is considered insignificant for this type of measurement. The glider platform thus appears ideal for the study of near-surface ocean turbulence in the presence of nonbreaking surface waves.

On the other hand, surface wave effects can have a significant influence on the estimated depth, and vertical velocity, of the glider. This is only expected to occur in shallow water wave fields, and is due to an incomplete cancellation of pressure anomalies arising from both the vertical excursion of the glider in the water column, and the pressure perturbation of the wave orbital acceleration. Observations taken in the shallow North Sea during a summer storm confirm the prediction of an increasing amplitude of this effect with depth. We propose that an effective remedy to this effect is to apply a low-pass filter to the pressure data. The filter should be chosen with a cutoff frequency low enough to remove the dominant frequency in the wave signal. The filtered depth reading then corresponds to a depth that is representative of an undisturbed water column, and provides a sensible coordinate to map the observations to.

Acknowledgments. We acknowledge financial support from the Helmholtz Association through the PACES II program, as well as the German Research Foundation (DFG). This paper is a contribution to the projects M6 and T2 of the Collaborative Research Centre TRR 181, "Energy Transfers in Atmosphere and Ocean" funded through DFG Grant 274762653.

Data availability statement. All datasets used herein are publicly available at the following sources: wave buoy data platform Gascogne Buoy, WMO6200001 at https://www.emodnetphysics.eu/Map/platinfo/piroosplot.aspx?platformid=1043915, model data German Bight at https://codm.hzg.de/codm/ dataset "wave model for the German Bight," and glider data North Sea 2019 at https://doi.org/10.5281/zenodo.4740627.

\section{APPENDIX}

\section{Linearization of $U^{2}$ and $\alpha$}

The glider flight model, as detailed by Eqs. (9) and (10), contains two independent parameters $U^{2}$ and $\alpha$. The perturbation approach employed in this work, favors the use of velocities $v_{x}$ and $v_{z}$. We write $v_{i}=v_{i 0}+v_{i 1}$, with $i \in\{x, z\}$. The indices 0 and 1 denote the steady-state and perturbed velocity, respectively. To express $U^{2}$ in steady-state parameters and the perturbed parameters $v_{x 1}$ and $v_{z 1}$, we write

$$
\begin{aligned}
U^{2} & =\left(v_{x 0}+v_{x 1}\right)^{2}+\left(v_{z 0}+v_{z 1}\right)^{2} \\
& \approx v_{x 0}^{2}+2 v_{x 0} v_{x 1}+v_{z 0}^{2}+2 v_{z 0} v_{z 1} \\
& =U_{0}^{2}+2\left(v_{x 0} v_{x 1}+v_{z 0} v_{z 1}\right),
\end{aligned}
$$

where it is assumed that $v_{i 1} \ll v_{i 0}$ with $i \in\{x, z\}$. The angle of attack is written as $\alpha=\alpha_{0}+\alpha_{1}$ and $\alpha_{1} \ll \alpha_{0}$. To express $\alpha_{1}$ in terms of perturbed velocities, we write

$$
\tan (\theta+\alpha)=\frac{v_{z 0}+v_{z 1}}{v_{x 0}+v_{x 1}} .
$$

Applying a Taylor expansion to the left-hand side of (A4), we get

$$
\begin{aligned}
\tan \left(\theta+\alpha_{0}\right)+[1 & \left.+\tan ^{2}\left(\theta+\alpha_{0}\right)\right] \alpha_{1}+O\left(\alpha_{1}^{2}\right)=\frac{v_{z 0}+v_{z 1}}{v_{x 0}+v_{x 1}} \\
& \Rightarrow \frac{v_{z 0}}{v_{x 0}}+\left[1+\frac{v_{z 0}^{2}}{v_{x 0}^{2}}\right] \alpha_{1} \approx \frac{v_{z 0}+v_{z 1}}{v_{x 0}+v_{x 1}} \\
& \Rightarrow \alpha_{1} \approx \frac{v_{x 0} v_{z 1}-v_{z 0} v_{x 1}}{U_{0}^{2}}
\end{aligned}
$$

so that

$$
\alpha \approx \alpha_{0}+\frac{v_{x 0} v_{z 1}-v_{z 0} v_{x 1}}{U_{0}^{2}}
$$

\section{REFERENCES}

Carpenter, J. R., A. Rodrigues, L. K. P. Schultze, L. M. Merckelbach, N. Suzuki, B. Baschek, and L. Umlauf, 2020: Shear instability and turbulence in a submesoscale front following a storm. Geophys. Res. Lett., 47, e2020GL090365, https://doi.org/ 10.1029/2020GL090365.

Dingemans, M. W., 1997: Water Wave Propagation over Uneven Bottoms: Part 1-Linear Wave Propagation. Advanced Series on Ocean Engineering, Vol. 13. World Scientific, $471 \mathrm{pp}$.

Fer, I., A. K. Peterson, and J. E. Ullgren, 2014: Microstructure measurements from and underwater glider in the turbulent Faroe Bank channel overflow. J. Atmos. Oceanic Technol., 31, 1128-1150, https://doi.org/10.1175/JTECHD-13-00221.1.

Kundu, P., I. Cohen, and H. Hu, 2004: Fluid Mechanics. 2nd ed. Elsevier, 759 pp.

Merckelbach, L., D. Smeed, and G. Griffiths, 2010: Vertical velocities from underwater gliders. J. Atmos. Oceanic Technol., 27, 547-563, https://doi.org/10.1175/2009JTECHO710.1.

— A. Berger, G. Krahmann, M. Dengler, and J. R. Carpenter, 2019: A dynamic flight model for Slocum gliders and implications for turbulence microstructure measurements. J. Atmos. Oceanic Technol., 36, 281-296, https://doi.org/ 10.1175/JTECH-D-18-0168.1.

Moldotsov, S., A. Annis, R. Amon, and P. Perez-Brunius, 2020: Turbulent mixing in a loop-current eddy from gliderbased microstructure observations. Geophys. Res. Lett., 47, e2020GL088033, https://doi.org/10.1029/2020GL088033.

Moum, J., M. Gregg, R. Lien, and M. Carr, 1995: Comparison of turbulence kinetic energy dissipation rate estimates from two ocean microstructure profilers. J. Atmos. Oceanic Technol., 
12, 346-366, https://doi.org/10.1175/1520-0426(1995)012<0346: COTKED $>2.0 . \mathrm{CO} ; 2$.

Newman, J., 1977: Marine Hydrodynamics. MIT Press, 402 pp.

Palmer, M., G. Stephenson, M. Inall, C. Balfour, A. Düsterhus, and J. Green, 2015: Turbulence and mixing by internal waves in the Celtic Sea determined from ocean glider microstructure measurements. J. Mar. Syst., 144, 57-69, https://doi.org/10.1016/ j.jmarsys.2014.11.005.

Scheifele, B., S. Waterman, L. Merckelbach, and J. R. Carpenter, 2018: Measuring the dissipation rate of turbulent kinetic energy in strongly stratified, low-energy environments: A case study from the Arctic Ocean. J. Geophys. Res. Oceans, 123, 5459-5480, https://doi.org/10.1029/2017JC013731.

Schultze, L. K. P., L. M. Merckelbach, and J. R. Carpenter, 2017: Turbulence and mixing in a shallow stratified shelf sea from underwater gliders. J. Geophys. Res. Oceans, 122, 9092-9109, https://doi.org/10.1002/2017JC012872.

,-- , and -2020 : Storm-induced turbulence alters shelf sea vertical fluxes. Limnol. Oceanogr. Lett., 5, 264-270, https:// doi.org/10.1002/lol2.10139.

St. Laurent, L., and S. Merrifield, 2017: Measurements of nearsurface turbulence and mixing from autonomous ocean gliders. Oceanography, 30 (2), 116-125, https://doi.org/10.5670/ oceanog.2017.231.
Staneva, J., A. Behrens, and N. Groll, 2014: Recent advances in wave modelling for the North Sea and German Bight. Küste, 81, 233-254.

- , - and K. Wahle, 2015: Wave modelling for the German Bight coastal-ocean predicting system. J. Phys. Conf. Ser., 633, 012117, https://doi.org/10.1088/1742-6596/633/1/012117.

Testor, P., and Coauthors, 2019: OceanGliders: A component of the integrated GOOS. Front. Mar. Sci., 6, 422, https://doi.org/ 10.3389/fmars.2019.00422.

Teutsch, I., R. Weisse, J. Moeller, and O. Krueger, 2020: A statistical analysis of rogue waves in the southern North Sea. Nat. Hazards Earth Syst. Sci., 20, 2665-2680, https://doi.org/10.5194/nhess-202665-2020.

Tucker, M. J., 1991: Waves in Ocean Engineering: Measurement, Analysis and Interpretation. Marine Science, Ellis Horwood, $431 \mathrm{pp}$.

Waterhouse, A., and Coauthors, 2014: Global patterns of diapycnal mixing from measurements of the turbulent dissipation rate. J. Phys. Oceanogr., 44, 1854-1872, https://doi.org/10.1175/ JPO-D-13-0104.1

Wijesekera, H., D. Wang, and E. Jarosz, 2020: Dynamics of the diurnal warm layer: Surface jet, high-frequency internal waves, and mixing. J. Phys. Oceanogr., 50, 2053-2070, https:// doi.org/10.1175/JPO-D-19-0285.1. 